Cahiers $d u$ MONDE RUSSE

\section{Cahiers du monde russe}

Russie - Empire russe - Union soviétique et États indépendants

$49 / 4 \mid 2008$

Destins individuels et terreur. Jeunesse dans la société post-stalinienne

\title{
Mary W. Cavender, Nests of the gentry
}

\section{Alessandro Stanziani}

\section{(2) OpenEdition}

Journals

Édition électronique

URL : https://journals.openedition.org/monderusse/6933

DOI : 10.4000/monderusse.6933

ISSN : $1777-5388$

Éditeur

Éditions de l'EHESS

Édition imprimée

Date de publication : 28 décembre 2008

Pagination : 732-734

ISBN : 978-2-7132-2197-2

ISSN : $1252-6576$

Référence électronique

Alessandro Stanziani, « Mary W. Cavender, Nests of the gentry ", Cahiers du monde russe [En ligne], 49/4 | 2008, mis en ligne le 23 décembre 2009, consulté le 04 septembre 2022. URL : http://

journals.openedition.org/monderusse/6933; DOI : https://doi.org/10.4000/monderusse.6933

Ce document a été généré automatiquement le 4 septembre 2022.

Tous droits réservés 


\title{
Mary W. Cavender, Nests of the gentry
}

\author{
Alessandro Stanziani
}

\section{RÉFÉRENCE}

Mary W. CAVENDER, Nests of the gentry. Family, estate and local loyalties in

Provincial Russia. Newark : University of Delaware Press, 2007.

1 Cet ouvrage a pour ambition de montrer qu'une certaine noblesse provinciale russe n'était guère " aliénée », c'est-à-dire peu intéressée à la gestion de ses domaines et à la vie locale, mais qui, au contraire, elle était fortement ancrée dans cette réalité locale dont elle participait activement à la vie non seulement économique, mais aussi sociale et religieuse. Afin de démontrer ce point, l'auteur étudie les comportements, les réseaux sociaux et les croyances de la noblesse de la province de Tver pendant la première moitié du XIX ${ }^{\mathrm{e}}$ siècle.

2 Le titre de l'ouvrage reprend celui d'un célèbre nouvelle de Turgenev, Dvorjanskoe gnezdo, que l'auteur passe au pluriel, afin de mettre en évidence la multiplicité des points d'ancrage dans la vie provinciale. Le premier chapitre étudie les liens à l'intérieur des familles nobles et entre famille, les manières dont les nobles se définissent et s'(auto)identifient, leurs stratégies de transmission des biens, sans oublier le rôle des femmes qui, sans être égalitaire à celui des hommes, n'est pas négligeable non plus au sein des ménages. L'auteur exploite des sources intéressantes et en bonne partie inédites, telles que la correspondance familiale et les registres généalogiques (rodoslovnye knigi). Ces documents mettent en évidence l'attachement de tous les membres d'une famille donnée à la vie locale. L'éducation des enfants, leurs années passées dans la capitale, à Moscou, voire même à l'étranger et leur retour à Tver sont étudiés dans les détails. Les filles reçoivent une éducation différente et ont également accès à des parts d'héritage autres que ceux des garçons. En particulier, elles reçoivent en dot, puis en héritage de 
l'argent et éventuellement des terres, mais sans bâtiments ni paysans. Les domaines habités sont en effet réservés aux garçons.

3 La correspondance, étudiée en détail, permet également de montrer l'importance que tous les membres de la famille attachent à l'harmonie de cette dernière. Ce sentiment apparait d'autant plus central qu'il ne cesse d'être ébranlé du fait des tensions en matière d'héritage, d'éducation, carrière et gestion du domaine, sans oublier les choix concernant les mariages. Dans ce contexte, le domaine apparaît comme davantage qu'une simple propriété ; c'est un lieu de souvenirs, d'émotions et sentiments qui ne cessent de jaillir des lettres découvertes par l'auteur.

Le deuxième chapitre précise le lien entre propriété et paternalisme ou, en d'autres mots, la manière dont la notion de propriété contribue à définir celle du «noble ». Selon cette perspective, la manière dont les membres de la famille se relatent au domaine et aux propriétés en général ne relève pas seulement du calcul économique (même si ce dernier joue un rôle important), mais répond aussi à des sentiments et des émotions. Selon cette perspective, le servage n'est envisagé ni du point de vue strictement économique de sa rentabilité ni de celui " éthique » en général. Les nobles de Tver justifient le servage à partir, précisément, de leur propre attachement et engagement dans leurs domaines locaux. Autrement dit, et contrairement aux arguments dominants dans l'historiographie, le servage se justifie moins par le "service» dû au tsar et par l'absentéisme des nobles que par leur présence et engagement dans les provinces. C'est là une des contributions majeures de cet ouvrage. Elle conduit l'auteur à détailler les interventions nombreuses des propriétaires nobles dans les activités des paysans, leurs intérêts dans la manufacture et la proto-industrie et, de manière générale, dans les activités commerciales. Intérêts économiques, attachement et loyauté à la vie locale interviennent ensemble à justifier ces attitudes.

5 Le troisième chapitre cherche à étaler les idéaux, la culture et les connaissances (y compris les connaissances techniques et scientifiques) des nobles de Tver. C'est probablement le chapitre le plus faible de l'ouvrage car il s'appuie en bonne partie sur des ouvrages généraux déjà disponibles sur cet aspect (Vucinich, Emmons et Confino parmi d'autres), sans ajouter grand-chose. Au contraire, il est même surprenant de trouver (p.124) des remarques sur l'incapacité des nobles de procéder à un véritable calcul économique rationnel, alors que Confino et, plus récemment, Melton et Hoch, ont mis en évidence la spécificité du calcul économique et de la rationalité des nobles. C'est une orientation d'autant plus surprenante qu'elle est en contradiction avec les affirmations des deux chapitres précédents.

6 Le quatrième chapitre reprend le fil des deux premiers chapitres en revenant sur la sociabilité et l'activité politique des familles nobles de Tver. Sa contribution la plus importante consiste à mettre en évidence les réseaux et les modalités de la sociabilité au sein de la vie locale ; ce qui permet de compléter les images sur la vie et les orientations politiques de la noblesse provinciale déjà connues par d'autres travaux.

7 Dans l'ensemble, il s'agit d'un ouvrage qui permet de mieux appréhender la vie et les orientations d'une certaine noblesse provinciale. Certes, Tver n'est pas une région quelconque: au xIX siècle elle connaît à plusieurs reprises des propriétaires locaux ouvertement engagés du côté des réformateurs. Les exilés politiques sont nombreux et, avec eux, les publications non seulement politiques, mais aussi sur la vie économique et sociale de la paysannerie locale. Ce qui donne à l'auteur l'avantage de pouvoir aisément croiser ses sources micro-historiques avec des analyses d'ensemble sur cette région. 
8 La question demeure cependant de ce que ces monographies ajoutent à notre connaissance de l'histoire russe. Sans doute, les images monolithiques que nous avons de la Russie en sortent-elles ébranlées; les diversités des conditions et de la vie locales enrichissent notre compréhension de l'ensemble. En même temps, ce genre de monographies a toujours été répandu, à l'époque tsariste (surtout pendant la seconde moitié du xix ${ }^{e}$ siècle) comme soviétique. La vie économique et sociale dans telle ou telle province a été étudiée de près et constitue un des traits forts de l'historiographie russosoviétique par rapport à celle que nous connaissons en plusieurs pays d'Europe « occidentale».

9 Cependant, ces monographies mènent souvent leurs auteurs à une position difficile à défendre : si, en effet, elles défendent la spécificité de la région étudiée, alors il devient difficile d'en tirer des conclusions plus générales. Si, au contraire, la monographie confirme les conclusions déjà obtenues par ailleurs, alors sa nécessité et valeur heuristique sont assez réduites. Ce n'est pas un hasard si, en ce cas aussi, l'auteur oscille entre ces deux positions, l'étude de Tver servant tantôt à confirmer tantôt à nier ce que nous connaissions déjà par ailleurs. En effet, il ne faudrait pas confondre ce genre de monographie avec les études de microhistoire proprement dite, qui, elles, se distinguent précisément par la remise en discussion radicale de l'argument de la représentativité du cas étudié. Au contraire, c'est la pertinence du cas par rapport aux questions posées qui est mis à l'avant. Or, cette approche n'est pas celle de Mary Cavender, dont la question principale se résout à savoir si la noblesse provinciale était attachée à la vie locale.

Ces considérations, n'enlèvent rien aux principaux mérites de cet ouvrage qui rajoute des éléments utiles, voire indispensables, pour tout travail de synthèse à venir sur la noblesse russe. Il contribue aussi à ébranler le mythe d'une noblesse de province absente et distante des réalités locales et permet de mieux comprendre l'attachement des élites russes à un système qui était loin de constituer la simple imposition des hauts dignitaires de la capitale. 